Proceedings of the Edinburgh Mathematical Society (2003) 46, 609-625 (C)

DOI:10.1017/S0013091503000336 Printed in the United Kingdom

\title{
ON THE OSCILLATION OF SELF-ADJOINT MATRIX HAMILTONIAN SYSTEMS
}

\author{
QIGUI YANG ${ }^{1}$ AND SUI SUN CHENG ${ }^{2}$ \\ ${ }^{1}$ Department of Mathematics, Tsinghua University, Beijing 100084, \\ People's Republic of China, and \\ Department of Mathematics, Guangxi Normal University, Guilin 541004, \\ People's Republic of China (yangqigui@263.net) \\ ${ }^{2}$ Department of Mathematics, Tsinghua University, Hsinchu, Taiwan 30043, \\ Republic of China (sscheng@math.nthu.edu.tw)
}

(Received 27 November 2002)

\begin{abstract}
By means of monotone functionals and positive linear functionals defined on suitable matrix spaces as well as new generalized Riccati transformations, oscillation criteria for self-adjoint linear Hamiltonian matrix systems are obtained. Our results are generalizations and improvements of many existing results.
\end{abstract}

Keywords: matrix Hamiltonian system; oscillation criterion; monotone functional; positive linear functional

2000 Mathematics subject classification: Primary 34A30; 34C10

\section{Introduction}

Self-adjoint linear Hamiltonian matrix systems arise in many dynamical problems and have been studied by many authors (see, for example, $[\mathbf{1}-\mathbf{1 8}])$. In this paper, we consider linear self-adjoint Hamiltonian matrix systems of the form

$$
\left.\begin{array}{rl}
U^{\prime}(x) & =A(x) U(x)+B(x) V(x) \\
V^{\prime}(x) & =C(x) U(x)-A^{*}(x) V(x)
\end{array}\right\}
$$

where $A(x), B(x)=B^{*}(x)>0$ and $C(x)=C^{*}(x)$ are $n \times n$ real continuous matrix functions on the interval $I=[a, \infty)$. Here and below, the transpose of the matrix $M$ is denoted by $M^{*}$ and its positive definiteness is denoted by $M>0$.

A solution $(U(x), V(x))$ of the system (1.1) is said to be non-trivial if $\operatorname{det} U(x) \neq 0$ for at least one $x \in[a, \infty)$, and a non-trivial solution $(U(x), V(x))$ of $(1.1)$ is said to be prepared or self-conjugate if

$$
U^{*}(x) V(x)-V^{*}(x) U(x)=0, \quad x \geqslant a .
$$


System (1.1) is said to be oscillatory on $[a, \infty)$ if there is a non-trivial prepared solution $(U(x), V(x))$ of $(1.1)$ such that $\operatorname{det} U(x)$ vanishes at least once on $[T, \infty)$ for each $T \geqslant a$. Otherwise, it is said to be non-oscillatory. It is well known [14, Theorem 8.1, p. 303] that if the system (1.1) is oscillatory, then every non-trivial prepared solution $(\bar{U}(x), \bar{V}(x))$ of (1.1) has the property that $\operatorname{det} \bar{U}(x)$ vanishes at least once on $[T, \infty)$ for every $T>a$.

Oscillatory properties of (1.1) as well as the special case

$$
\left.\begin{array}{l}
U^{\prime}(x)=A(x) U(x)+E_{n}(x) V(x), \\
V^{\prime}(x)=C(x) U(x)-A^{*}(x) V(x),
\end{array}\right\}
$$

where $E_{n}$ is the $n \times n$ identity matrix, are important in optimization of certain functionals associated with (1.1). Therefore, such properties have been studied quite extensively. In particular, special cases of (1.1), such as

$$
\left(P(x) U^{\prime}(x)\right)^{\prime}+Q(x) U(x)=0
$$

and

$$
U^{\prime \prime}(x)+Q(x) U(x)=0,
$$

have been studied and many results related to oscillation obtained (see, for example, $[\mathbf{1}-\mathbf{3}, \mathbf{5}, \mathbf{8}, \mathbf{1 1}, \mathbf{1 5}, \mathbf{1 8}]$ and the references quoted therein).

To motivate what follows, let us go through some of the well-known contributions. First of all, for the scalar equation (as a special case of (1.4)),

$$
\left(p(x) y^{\prime}(x)\right)^{\prime}+q(x) y(x)=0 .
$$

Leighton's criterion (see [9]) states that (1.6) is oscillatory on the interval $[0, \infty)$ if

$$
\int_{0}^{\infty} p^{-1}(t) \mathrm{d} t=\infty \text { and } \int_{0}^{\infty} q(t) \mathrm{d} t=\infty .
$$

In 1949, Wintner [16] showed that

$$
y^{\prime \prime}(x)+q(x) y(x)=0
$$

is oscillatory if

$$
\lim _{t \rightarrow \infty} \frac{1}{t} \int_{0}^{t}\left(\int_{0}^{s} q(u) \mathrm{d} u\right) \mathrm{d} s=\infty .
$$

In a different direction, in 1952 Hartman [4] showed that (1.7) is oscillatory when

$$
-\infty<\liminf _{t \rightarrow \infty} \frac{1}{t} \int_{0}^{t}\left(\int_{0}^{s} q(u) \mathrm{d} u\right) \mathrm{d} s<\limsup _{t \rightarrow \infty} \frac{1}{t} \int_{0}^{t}\left(\int_{0}^{s} q(u) \mathrm{d} u\right) \mathrm{d} s \leqslant \infty .
$$

In 1978, another important type of criterion was given by Kamenev [7], who showed that if for some positive integer $m>2$,

$$
\limsup _{t \rightarrow \infty} \frac{1}{t^{m-1}} \int_{0}^{t}(t-s)^{m-1} q(s) \mathrm{d} s=\infty,
$$

then Equation (1.7) is oscillatory. 
Oscillation results based on a Kamenev-type criterion for (1.7) can be found in earlier papers of Philos [12], Li [10], Wong [17] and other references cited therein.

Kamenev's Theorem has also been extended by Philos [13] for (1.7) using the general means method, which was further extended to the matrix differential system (1.4) by Erbe et al. $[\mathbf{3}]$ as follows.

Theorem A. Let $H(t, s)$ and $h(t, s)$ be continuous on $D=\left\{(t, s) \mid t \geqslant s \geqslant t_{0}\right\}$ such that $H(t, t)=0$ for $t \geqslant t_{0}$ and $H(t, s)>0$ for $t>s \geqslant t_{0}$. Suppose further that the partial derivative $\partial H(t, s) / \partial s$ is non-positive and continuous for $t \geqslant s \geqslant t_{0}$, and $h(t, s)$ is defined by

$$
\partial H(t, s) / \partial s=-h(t, s) H^{1 / 2}(t, s),(t, s) \in D .
$$

If

$$
\limsup _{t \rightarrow \infty} \frac{1}{H\left(t, t_{0}\right)} \lambda_{\max }\left[\int_{t_{0}}^{t}\left(H(t, s) Q(s)-\frac{1}{4} h^{2}(t, s) P(s)\right) \mathrm{d} s\right]=\infty
$$

where $\lambda_{\max }(A)$ stands for the maximal eigenvalue of the matrix $A$, then (1.4) is oscillatory.

In 1998, Meng et al. [11] employed the idea of generalized Riccati transformation to obtain an oscillation criterion for (1.4) and extended an earlier result of Erbe et al. [3]. Their work was subsequently generalized by Wang [15] in 2001 .

In 2000, Kumari and Umamaheswaram [8] obtained oscillation theorems for (1.1) that extend that of Wintner [16] and Kamenev [7]. Other oscillation results based on a Wintner-type criterion for (1.1) and the special system (1.4) can also be found in a recent paper of the first author [18] and some of the references cited therein.

There are many other oscillation criteria. In view of this fact, it is therefore of interest to find unified results.

In $\S 2$ we approach our goal by using monotone functionals and new generalized Riccati transformations as well as considering functions $H(x, s) k(s)$, where $H(t, s)$ may have non-positive partial derivatives on $D_{0}=\{(x, s): x>s \geqslant a\}$ with respect to the second variable. We obtain Kamenev-type oscillation criteria (Theorems 2.2, 2.8, Corollaries 2.4-2.7) for the system (1.1) which include results of Kumari et al. [8, Theorems 2.3-2.7, 2.9], Meng et al. [11, Theorem 1], Erbe et al. [3, Theorems 1-7] and Wang [15, Theorems 1-6].

In $\S 3$ we present a set of new oscillation criteria by using positive linear functionals. The subsequent results are generalizations of the analogous oscillation criterion of Kumari and Umamaheswaram [8, Theorems 2.3, 2.9] for the system (1.1) and are generalizations and improvements of $\mathrm{Li}[\mathbf{1 0}]$, Philos [13] for the special case (1.6), and Kamenev [7] for (1.7).

Our results obtained in the above manner extend, improve and unify a number of existing results. In the final section, several examples that dwell upon the importance of our results are presented.

Throughout this paper we use the notation

$$
D_{0}=\{(x, s): 0 \leqslant a \leqslant s<x<\infty\} \quad \text { and } \quad D=\{(x, s): 0 \leqslant a \leqslant s \leqslant x<\infty\} .
$$




\section{Oscillation criteria in terms of monotone functionals}

Throughout this section we denote the eigenvalues of an $n \times n$ Hermitian matrix $A$ by $\lambda_{1}[A], \lambda_{2}[A], \ldots, \lambda_{n}[A]$, where $\lambda_{\min }[A]=\lambda_{n}[A] \leqslant \cdots \leqslant \lambda_{2}[A] \leqslant \lambda_{1}[A]=\lambda_{\max }[A]$.

In what follows, $\mathcal{S}$ will denote the linear space of all $n \times n$ real symmetric matrices endowed with the usual operations.

Definition 2.1. A functional $p: \mathcal{S} \rightarrow \mathbb{R}$ is said to be subhomogeneous if $p(\lambda K) \leqslant$ $\lambda p(K)$ whenever $K \in \mathcal{S}$ and $\lambda \geqslant 0$. Such a functional is said to be monotone (or nondecreasing) if $J-K \geqslant 0$ implies $p(J) \geqslant p(K)$ for $J, K \in \mathcal{S}$.

The first part of Definition 2.1 is found in Hartman [5, p. 328]. Note that because of the classical minimal characterization of the eigenvalues of a matrix in $\mathcal{S}$, the functional $p(K)=\lambda_{\max }(K)$ is monotone and subhomogeneous and is traditionally called the 'eigenvalue' functional. On the other hand, it is readily verified that if $P \geqslant 0$ in $\mathcal{S}$, then the nonlinear functional $p(K)=\lambda_{\max }(K+P)$ is also monotone and $\lambda_{\max }[K+P] \geqslant \lambda_{\max }[K]$. Furthermore, it is easy to see that the nonlinear trace functional on $\mathcal{S}$ defined by $p(K)=\operatorname{tr}\left(K+E_{n}\right)$ is also monotone.

Theorem 2.2. Let functions $H \in C(D ; \mathbb{R}), h \in C\left(D_{0} ; \mathbb{R}\right), \phi \in C^{1}([a, \infty) ; \mathbb{R}), k$ and $\beta \in C^{1}([a, \infty) ;(0, \infty))$ satisfy the following conditions:

$\left(H_{1}\right) H(x, x)=0$ for $x \geqslant a$ and $H(x, s)>0$ on $D_{0}$;

$\left(H_{2}\right) H$ has a continuous and non-positive partial derivative on $D_{0}$ with respect to the second variable;

$\left(H_{3}\right)-\frac{\partial}{\partial s}(H(x, s) k(s))-H(x, s) k(s) \frac{\beta^{\prime}(s)}{\beta(s)}=h(x, s)$ for all $(x, s) \in D_{0}$.

Suppose there exists a monotone subhomogeneous functional $p$ on $\mathcal{S}$ such that

$$
\limsup _{x \rightarrow \infty} \frac{1}{H(x, a)} p\left[\int_{a}^{x}\left\{H(x, s) k(s) T(s)+J_{0}(x, s)\right\} \mathrm{d} s\right]=\infty,
$$

where

$$
\begin{aligned}
J_{0}(x, s)= & H(x, s) k(s) v(s) \beta(s)\left[\phi\left(A+A^{*}\right)-A^{*} B^{-1} A\right](s) \\
& -v(s) \beta(s)\left[\frac{1}{2} h(x, s)+H(x, s) k(s) \phi(s)\right]\left[A^{*} B^{-1}+B^{-1} A\right](s) \\
& -v(s) \beta(s)\left[\left\{\frac{1}{2}[H(x, s) k(s)]^{-1 / 2} h(x, s)+[H(x, s) k(s)]^{1 / 2} \phi(s)\right\} B^{-1 / 2}(s)\right. \\
& \left.-[H(x, s) k(s)]^{1 / 2} \phi(s) B^{1 / 2}(s)\right]^{2} \\
v(x)= & \exp \left\{-2 \int_{a}^{x} \phi(s) \mathrm{d} s\right\}
\end{aligned}
$$

and

$$
T(x)=v(x) \beta(x)\left[-C-\phi\left(A^{*}+A\right)+\phi^{2} B-\phi^{\prime} E_{n}\right](x) .
$$

Then the system (1.1) is oscillatory. 
Proof. Suppose to the contrary that there exists a prepared solution $(U(x), V(x))$ of the system (1.1) which is not oscillatory. Without loss of generality, we may assume that $\operatorname{det} U(x) \neq 0$ for $x \geqslant a$. Define the matrix function $W(x)$ on $[a, \infty)$ by

$$
W(x)=\beta(x) v(x)\left[V(x) U^{-1}(x)+\phi(x) E_{n}\right]=\beta(x) Z(x) .
$$

From (1.2), it follows that

$$
\begin{aligned}
W^{*}(x) & =\beta(x) v(x)\left\{\left[U^{-1}(x)\right]^{*} V^{*}(x)+\phi(x) E_{n}\right\} \\
& =\beta(x) v(x)\left\{\left[U^{*}(x)\right]^{-1} U^{*}(x) V(x) U^{-1}(x)+\phi(x) E_{n}\right\}=W(x),
\end{aligned}
$$

which implies that $W$ is Hermitian. Differentiating the Hermitian matrix (2.5) and invoking (1.1), we have

$$
\begin{aligned}
W^{\prime}(x)= & \beta^{\prime}(x) Z(x)+\beta(x) Z^{\prime}(x) \\
= & \left(\frac{\beta^{\prime}}{\beta} W\right)(x)+\beta(x)\left\{-\left[A^{*} Z+Z A+\frac{1}{v} Z B Z\right](x)\right. \\
& \left.\quad+(\phi[Z B+B Z-2 Z])(x)-\left(\frac{1}{\beta} T\right)(x)\right\} \\
= & \left(\frac{\beta^{\prime}}{\beta} W\right)(x)-\left[\frac{1}{\beta v} W B W+A^{*} W+W A-\phi(W B+B W-2 W)\right](x)-T(x),
\end{aligned}
$$

for $x \in[a, \infty)$, where

$$
T(x)=\beta(x) v(x)\left(-C-\phi\left[A+A^{*}\right]+\phi^{2} B-\phi^{\prime} E_{n}\right)(x) .
$$

Replacing $x$ by $s$ in (2.6) and then multiplying the subsequent equation by $H(x, s) k(s)$ and integrating with respect to $s$ from $a$ to $x(x \geqslant a)$, and after some simple computation, we have

$$
\begin{aligned}
\int_{a}^{x} H(x, s) k(s) v(s) T(s) \mathrm{d} s \\
=-\int_{a}^{x} H(x, s) k(s) W^{\prime}(s) \mathrm{d} s+\int_{a}^{x} H(x, s) k(s) \frac{\beta^{\prime}(s)}{\beta(s)} W(s) \mathrm{d} s \\
\quad-\quad \int_{a}^{x} H(x, s) k(s)\left[\frac{1}{\beta v} W B W+A^{*} W+W A-\phi(W B+B W-2 W)\right](s) \mathrm{d} s \\
=H(x, a) k(a) W(a)-\int_{a}^{x}\left[-\frac{\partial}{\partial s}(H(x, s) k(s))-H(x, s) k(s) \frac{\beta^{\prime}(s)}{\beta(s)}\right] W(s) \mathrm{d} s \\
\quad-\int_{a}^{x} H(x, s) k(s)\left[\frac{1}{\beta v} W B W+A^{*} W+W A-\phi(W B+B W-2 W)\right](s) \mathrm{d} s \\
=H(x, a) k(a) W(a)-\int_{a}^{x}[h(x, s)+2 \phi(s) H(x, s) k(s)] W(s) \mathrm{d} s \\
\quad-\int_{a}^{x} H(x, s) k(s)\left[\frac{1}{\beta v} W B W+A^{*} W+W A-\phi(W B+B W)\right](s) \mathrm{d} s \\
=H(x, a) k(a) W(a)-\int_{a}^{x} R^{-1}(s) G(x, s) R^{-1}(s) \mathrm{d} s
\end{aligned}
$$


where

$$
R(x)=\left[\frac{1}{\beta(x) v(x)} B(x)\right]^{1 / 2}
$$

and

$$
\begin{gathered}
G(x, s)=H(x, s) k(s)\{[R W R][R W R]\}(s)+[h(x, s)+2 \phi(s) H(x, s) k(s)](R W R)(s) \\
+H(x, s) k(s)\left\{R\left[A^{*} W+W A-\phi(W B+B W)\right] R\right\}(s) .
\end{gathered}
$$

Let

$$
\begin{aligned}
Q(x, s)=[H(x, s) k(s)]^{1 / 2}\left[R W-\phi \beta v R+R^{-1} A\right](s) \\
+\left\{\frac{1}{2}[H(x, s) k(s)]^{-1 / 2} h(x, s)+[H(x, s) k(s)]^{1 / 2} \phi(s)\right\} R^{-1}(s) .
\end{aligned}
$$

Then

$$
\begin{aligned}
Q^{*}(x, s)=[H(x, s) k(s)]^{1 / 2}\left[W R-\phi \beta v R+A^{*} R^{-1}\right](s) \\
+\left\{\frac{1}{2}[H(x, s) k(s)]^{-1 / 2} h(x, s)+[H(x, s) k(s)]^{1 / 2} \phi(s)\right\} R^{-1}(s) .
\end{aligned}
$$

Note that

$$
\begin{aligned}
& {[h(x, s)+2 \phi(s) H(x, s) k(s)] W(s)} \\
& +H(x, s) k(s)\left[\frac{1}{\beta v} W B W+A^{*} W+W A-\phi(W B+B W)\right](s) \\
& =R^{-1}(s) G(x, s) R^{-1}(s) \\
& =\left(Q^{*} Q\right)(x, s)+H(x, s) k(s) v(s) \beta(s)\left[\phi\left(A+A^{*}\right)-A^{*} B^{-1} A\right](s) \\
& \quad-v(s) \beta(s)\left[\frac{1}{2} h(x, s)+H(x, s) k(s) \phi(s)\right]\left[A^{*} B^{-1}+B^{-1} A\right](s) \\
& \quad-v(s) \beta(s)\left[\left\{\frac{1}{2}(H(x, s) k(s))^{-1 / 2} h(x, s)+(H(x, s) k(s))^{1 / 2} \phi(s)\right\} B^{-1 / 2}(s)\right. \\
& \left.\quad-(H(x, s) k(s))^{1 / 2} \phi(s) B^{1 / 2}(s)\right]^{2} \\
& =\left(Q^{*} Q\right)(x, s)+J_{0}(x, s) .
\end{aligned}
$$

By (2.7) and (2.8), we obtain

$$
\int_{a}^{x} H(x, s) k(s) T(s) \mathrm{d} s=H(x, a) k(a) W(a)-\int_{a}^{x}\left\{\left(Q^{*} Q\right)(x, s)+J_{0}(x, s)\right\} \mathrm{d} s,
$$

which implies

$$
\begin{aligned}
\int_{a}^{x}\left\{H(x, s) k(s) T(s)+J_{0}(x, s)\right\} \mathrm{d} s & =H(x, a) k(a) W(a)-\int_{a}^{x}\left(Q^{*} Q\right)(x, s) \mathrm{d} s \\
& \leqslant H(x, a) k(a) W(a) .
\end{aligned}
$$

Hence, from Definition 2.1 of a monotone subhomogeneous functional $p$ on $\mathcal{S}$, we have

$$
p\left[\int_{a}^{x}\left\{H(x, s) k(s) T(s)+J_{0}(x, s)\right\} \mathrm{d} s\right] \leqslant p[H(x, a) k(a) W(a)],
$$


for $x>a$, and

$$
\frac{1}{H(x, a)} p\left[\int_{a}^{x}\left\{H(x, s) k(s) T(s)+J_{0}(x, s)\right\} \mathrm{d} s\right] \leqslant p[k(a) W(a)]<\infty,
$$

which is contrary to $(2.1)$. The proof is complete.

Remark 2.3. Assume that $K, P \in \mathcal{S}$ and $P>0$. Let $p(K)$ be $\lambda_{\max }[K]$ in Theorem 2.2 with the other conditions unchanged, then the system (1.1) is oscillatory.

Furthermore, in Theorem 2.2, suppose in addition that $\liminf _{t \rightarrow \infty} H(t, s) \geqslant 1$ for sufficiently large $s \in \mathbb{R}$. Let the monotone subhomogeneous functional $p$ on $\mathcal{S}$ be replaced by a monotone functional $q: \mathcal{S} \rightarrow \mathbb{R}$ satisfying $q(\lambda K) \leqslant \lambda q(K)$ whenever $K \in \mathcal{S}$ and $\lambda \geqslant 1$, while the other conditions of Theorem 2.2 are kept unchanged. Then the system (1.1) is oscillatory. In fact, the proof of Theorem 2.2 may be easily modified to yield a proof of the new statement. Note that $q(K)$ may be chosen as $\lambda_{\max }[K+P]$ or $\operatorname{tr}\left(K+E_{n}\right)$.

Let $p(K)=\lambda_{\max }[K]$ and $\phi(t) \equiv 0$ in Theorem 2.2, then the following corollary is an extension of Theorem 2.3 of Kumari and Umamaheswaram [8], Theorem 1 of Erbe et al. [3] and Theorem 3 of Wang [15].

Corollary 2.4. Let functions $H \in C(D ; \mathbb{R}), h \in C\left(D_{0} ; \mathbb{R}\right), k, \beta \in C^{1}([a, \infty) ;(0, \infty))$ satisfy conditions $\left(H_{1}\right)-\left(H_{3}\right)$ of Theorem 2.2 .

If

$$
\limsup _{x \rightarrow \infty} \frac{1}{H(x, a)} \lambda_{1}\left[J_{0}(x)\right]=\infty
$$

where

$$
\begin{aligned}
& J_{0}(x)=-\int_{a}^{x} \beta(s)\{H(x, s) k(s)\left[C+A^{*} B^{-1} A\right](s)+\frac{1}{2} h(x, s) \\
&\left.\times\left[A^{*} B^{-1}+B^{-1} A\right](s)+\frac{h^{2}(x, s)}{4 H(x, s) k(s)} B^{-1}(s)\right\} \mathrm{d} s,
\end{aligned}
$$

then the system (1.1) is oscillatory.

Corollary 2.5. Let functions $H \in C(D ; \mathbb{R}), h \in C\left(D_{0} ; \mathbb{R}\right), k, \beta \in C^{1}([a, \infty) ;(0, \infty))$ and $J_{0}(x)$ be as in Corollary 2.4 and let $G_{1}(x)$ be the matrix-valued function

$$
G_{1}(x)=J_{0}(x)+\int_{a}^{x} \frac{h^{2}(x, s)}{4 H(x, s) k(s)} \beta(s) B^{-1}(s) \mathrm{d} s .
$$

(I) If

$$
\limsup _{x \rightarrow \infty} \frac{1}{H(x, a)} \lambda_{1}\left[G_{1}(x)\right]=\infty
$$

and

$$
\limsup _{x \rightarrow \infty} \frac{1}{H(x, a)} \lambda_{1}\left[\int_{a}^{x} \frac{h^{2}(x, s)}{H(x, s) k(s)} \beta(s) B^{-1}(s) \mathrm{d} s\right]<\infty,
$$

then the system (1.1) is oscillatory on $[a, \infty)$. 
(II) If $B(x)=\operatorname{diag}\left(b_{1}(x), b_{2}(x), \ldots, b_{n}(x)\right)$, where the $b_{i}(x)$ are continuous and positive for $x \geqslant a$ and $b(x)=\min _{1 \leqslant i \leqslant n} b_{i}(x)$, then the same conclusion as in (I) holds if condition (2.13) is replaced by the condition

$$
\limsup _{x \rightarrow \infty} \frac{1}{H(x, a)} \int_{a}^{x} \frac{h^{2}(x, s) \beta(s)}{H(x, s) k(s) b(s)} \mathrm{d} s<\infty .
$$

(III) If $B(x)=E_{n}$ and the conditions (2.12) and (2.14) are, respectively, replaced by the conditions

$$
\begin{aligned}
\limsup _{x \rightarrow \infty} \frac{1}{H(x, a)} \lambda_{1}\left[-\int_{a}^{x} \beta(s)\{H(x, s) k(s)[C\right. & \left.+A^{*} A\right](s) \\
& \left.\left.+\frac{1}{2} h(x, s)\left[A^{*}+A\right](s)\right\} \mathrm{d} s\right]=\infty
\end{aligned}
$$

and

$$
\limsup _{x \rightarrow \infty} \frac{1}{H(x, a)} \int_{a}^{x} \frac{h^{2}(x, s)}{H(x, s) k(s)} \beta(s) \mathrm{d} s<\infty,
$$

then the system (1.3) is oscillatory on $[a, \infty)$.

The next lemma is a statement of Weyl's inequality, which appears in $[\mathbf{6}]$ and $[\mathbf{8}]$.

Lemma 2.6. Let $P, Q$ be $n \times n$ Hermitian matrices. Then

$$
\lambda_{i}[P]+\lambda_{n}[Q] \leqslant \lambda_{i}[P+Q] \leqslant \lambda_{i}[P]+\lambda_{1}[Q]
$$

for $i=1,2, \ldots, n$.

Proof of Corollary 2.5. By the definition of $G_{1}(x)$ and Lemma 2.6, we obtain

$$
\begin{aligned}
\lambda_{1}\left[J_{0}(x)\right] & \geqslant \lambda_{1}\left[G_{1}(x)\right]+\lambda_{n}\left[-\int_{a}^{x} \frac{h^{2}(x, s)}{H(x, s) k(s)} \beta(s) B^{-1}(s) \mathrm{d} s\right] \\
& =\lambda_{1}\left[G_{1}(x)\right]-\lambda_{1}\left[\int_{a}^{x} \frac{h^{2}(x, s)}{H(x, s) k(s)} \beta(s) B^{-1}(s) \mathrm{d} s\right] \\
& \geqslant \lambda_{1}\left[G_{1}(x)\right]-\limsup _{x \rightarrow \infty} \lambda_{1}\left[\int_{a}^{x} \frac{h^{2}(x, s)}{H(x, s) k(s)} \beta(s) B^{-1}(s) \mathrm{d} s\right]-1
\end{aligned}
$$

for sufficiently large $x$. From (2.12) and (2.13), we have

$$
\limsup _{x \rightarrow \infty} \frac{1}{H(x, a)} \lambda_{1}\left[J_{0}(x)\right]=\infty .
$$

Hence (1.1) is oscillatory on $[a, \infty)$.

The second and third parts are consequences of the first. The proof is complete.

Choose $p(K)=\lambda_{\max }[K]$ and $\beta(t) \equiv 1$ in Theorem 2.2, then the following corollary is an extension of Theorem 2.9 of Kumari and Umamaheswaram [8], Theorem 1 of Meng et al. [11], Theorem 1 of Wang [15] and $\mathrm{Li}[\mathbf{1 0}]$. 
Corollary 2.7. Let functions $H \in C(D ; \mathbb{R})$ satisfy conditions $\left(H_{1}\right)$ and $\left(H_{2}\right)$ in Theorem 2.2. If there exist functions $\phi \in C^{1}([0, \infty),(0, \infty)), h \in C\left(D_{0} ; \mathbb{R}\right)$, and $k \in C^{1}([a, \infty) ;(0, \infty))$ such that

$$
\begin{gathered}
-\frac{\partial}{\partial s}(H(x, s) k(s))=h(x, s), \quad \forall(x, s) \in D_{0}, \\
\limsup _{x \rightarrow \infty} \frac{1}{H(x, a)} \lambda_{1}\left[\int_{a}^{x}\left\{H(x, s) k(s) T(s)+J^{0}(x, s)\right\} \mathrm{d} s\right]=\infty,
\end{gathered}
$$

where

$$
\begin{aligned}
J^{0}(x, s)= & H(x, s) k(s) v(s)\left[\phi\left(A+A^{*}\right)-A^{*} B^{-1} A\right](s) \\
& -v(s)\left[\frac{1}{2} h(x, s)+H(x, s) k(s) \phi(s)\right]\left[A^{*} B^{-1}+B^{-1} A\right](s) \\
& -v(s)\left[\left\{\frac{1}{2}[H(x, s) k(s)]^{-1 / 2} h(x, s)+[H(x, s) k(s)]^{1 / 2} \phi(s)\right\} B^{-1 / 2}(s)\right. \\
& \left.-[H(x, s) k(s)]^{1 / 2} \phi(s) B^{1 / 2}(s)\right]^{2}, \\
v(x)= & \exp \left\{-2 \int_{a}^{x} \phi(s) \mathrm{d} s\right\},
\end{aligned}
$$

and $T(s)=v(s)\left[-C-\phi\left(A^{*}+A\right)+\phi^{2} B-\phi^{\prime} E_{n}\right](s)$, then the system (1.1) is oscillatory.

If $h(t, s)$ is replaced by $h(t, s) \sqrt{H(t, s) k(s)}$ in Theorem 2.2, we have the following result. The proof is similar, so we omit the details.

Theorem 2.8. Let functions $H \in C(D ; \mathbb{R}), h \in C\left(D_{0} ; \mathbb{R}\right), \phi \in C^{1}([a, \infty) ; \mathbb{R}), k$ and $\beta \in C^{1}([a, \infty) ;(0, \infty))$ satisfy the conditions $\left(H_{1}\right)$ and $\left(H_{2}\right)$ in Theorem 2.2 and

$\left(H_{3}\right)-\frac{\partial}{\partial s}(H(x, s) k(s))-H(x, s) k(s) \frac{\beta^{\prime}(s)}{\beta(s)}=h(x, s) \sqrt{H(x, s) k(s)}$ for all $(x, s) \in D_{0}$.

Suppose there exists a monotone subhomogeneous functional $p$ on $\mathcal{S}$ such that

$$
\limsup _{x \rightarrow \infty} \frac{1}{H(x, a)} p\left[\int_{a}^{x}\left\{H(x, s) k(s) T(s)+J_{0}^{0}(x, s)\right\} \mathrm{d} s\right]=\infty,
$$

where

$$
\begin{aligned}
J_{0}^{0}(x, s)= & H(x, s) k(s) \beta(s) v(s)\left[\phi\left(A+A^{*}\right)-A^{*} B^{-1} A\right](s) \\
& -v(s) \beta(s)\left[\frac{1}{2} h(x, s) \sqrt{H(x, s) k(s)}+H(x, s) k(s) \phi(s)\right]\left[A^{*} B^{-1}+B^{-1} A\right](s) \\
& -v(s) \beta(s)\left[\left\{\frac{1}{2} h(x, s)+[H(x, s) k(s)]^{1 / 2} \phi(s)\right\} B^{-1 / 2}(s)\right. \\
& \left.-[H(x, s) k(s)]^{1 / 2} \phi(s) B^{1 / 2}(s)\right]^{2}, \\
v(x)= & \exp \left\{-2 \int_{a}^{x} \phi(s) \mathrm{d} s\right\},
\end{aligned}
$$

and $T(x)$ is defined in (2.4). Then the system (1.1) is oscillatory. 
We remark that corollaries similar to Corollaries 2.4-2.7 can be obtained by replacing $h(t, s)$ by $h(t, s) \sqrt{H(t, s) k(s)}$ and choosing the correct functionals in Theorem 2.8. Their statements are skipped since no new principles are involved.

Remark 2.9. Theorem 2.2 improves and extends the criteria in Kumari and Umamaheswaram [8, Theorem 2.3-2.9], Meng et al. [11], Erbe et al. [3] and Wang [15]. If $n=1$ and $A(x)=0$, the system (1.1) reduces to the scalar system (1.6). Note further that if we take $k(x) \equiv \beta(x) \equiv 1$ and $\phi(x) \equiv 0$, Corollaries 2.4-2.7 become Theorem 1 of Philos [12]

\section{Oscillation theorems in terms of positive linear functionals}

In this section, we establish several new oscillation criteria in terms of positive linear functionals.

Definition 3.1 (see [5]). A linear functional $L: \mathcal{S} \rightarrow \mathbb{R}$ satisfying

$$
L(K+J)=L(K)+L(J), \quad L(\lambda K)=\lambda L(K),
$$

for $K, J \in \mathcal{S}, \lambda \in \mathbb{R}$, is said to be 'positive' if $L(K)>0$ whenever $K \in \mathcal{S}$ and $K>0$.

Theorem 3.2. Let functions $H \in C(D ; \mathbb{R}), h \in C\left(D_{0} ; \mathbb{R}\right), \phi \in C^{1}([a, \infty) ; \mathbb{R}), k$ and $\beta \in C^{1}([a, \infty) ;(0, \infty))$ satisfy the following conditions:

$\left(H_{1}\right) H(x, x)=0$ for $x \geqslant a$ and $H(x, s)>0$ on $D_{0}$;

$\left(H_{2}\right) H$ has a continuous and non-positive partial derivative on $D_{0}$ with respect to the second variable;

$\left(H_{3}\right)-\frac{\partial}{\partial s}(H(x, s) k(s))-H(x, s) k(s) \frac{\beta^{\prime}(s)}{\beta(s)}=h(x, s)$ for all $(x, s) \in D_{0}$.

Suppose that there exists a linear positive functional $\mathrm{L}$ on $\mathcal{S}$ such that

$$
\limsup _{x \rightarrow \infty} \frac{1}{H(x, a)} \mathrm{L}\left[\int_{a}^{x}\left\{H(x, s) k(s) T(s)+J_{0}(x, s)\right\} \mathrm{d} s\right]=\infty,
$$

where $J_{0}(x, s), v(x)$ and $T(s)$ are defined by (2.2), (2.3) and (2.4), respectively. Then the system (1.1) is oscillatory.

Proof. Suppose to the contrary that there exists a prepared solution $(U(x), V(x))$ of the system (1.1) which is not oscillatory. Without loss of generality, we may assume that $\operatorname{det} U(x) \neq 0$ for $x \geqslant a$. Define the matrix function $W(x)$ on $[a, \infty)$ by $(2.5)$. As in the proof of Theorem 2.2, we can obtain (2.6) and (2.9). Moreover, it follows from (2.9) that

$$
\mathrm{L}\left[\int_{a}^{x}\left\{H(x, s) k(s) T(s)+J_{0}(x, s)\right\} \mathrm{d} s\right] \leqslant \mathrm{L}[H(x, a) k(a) W(a)], \quad x>a,
$$

yielding

$$
\frac{1}{H(x, a)} \mathrm{L}\left[\int_{a}^{x}\left\{H(x, s) k(s) T(s)+J_{0}(x, s)\right\} \mathrm{d} s\right] \leqslant \mathrm{L}[k(a) W(a)]<\infty,
$$

which is contrary to (3.1). The proof is complete. 
Let $\phi(t) \equiv 0$ in Theorem 3.2, then we get the following result.

Corollary 3.3. Let functions $H \in C(D ; \mathbb{R}), h \in C\left(D_{0} ; \mathbb{R}\right), k, \beta \in C^{1}([a, \infty) ;(0, \infty))$ satisfy conditions $\left(H_{1}\right)-\left(H_{3}\right)$ of Theorem 3.2.

If

$$
\limsup _{x \rightarrow \infty} \frac{1}{H(x, a)} \mathrm{L}\left[J_{0}(x)\right]=\infty,
$$

where $J_{0}(x)$ is defined as in (2.11), then the system (1.1) is oscillatory.

Corollary 3.4. Let functions $H \in C(D ; \mathbb{R}), h \in C\left(D_{0} ; \mathbb{R}\right), k, \beta \in C^{1}([a, \infty) ;(0, \infty))$ and $J_{0}(x)$ be as in Corollary 3.3 and let $G_{1}(x)$ be the matrix-valued function

$$
G_{1}(x)=J_{0}(x)+\int_{a}^{x} \frac{h^{2}(x, s)}{4 H(x, s) k(s)} \beta(s) B^{-1}(s) \mathrm{d} s .
$$

(I) If

$$
\limsup _{x \rightarrow \infty} \frac{1}{H(x, a)} \mathrm{L}\left[G_{1}(x)\right]=\infty
$$

and

$$
\limsup _{x \rightarrow \infty} \frac{1}{H(x, a)} \mathrm{L}\left[\int_{a}^{x} \frac{h^{2}(x, s)}{H(x, s) k(s)} \beta(s) B^{-1}(s) \mathrm{d} s\right]<\infty,
$$

then the system (1.1) is oscillatory on $[a, \infty)$.

(II) If $B(x)=\operatorname{diag}\left(b_{1}(x), b_{2}(x), \ldots, b_{n}(x)\right)$, where the $b_{i}(x)$ are continuous and positive for $x \geqslant a$ and $b(x)=\min _{1 \leqslant i \leqslant n} b_{i}(x)$, then the same conclusion as in (I) holds if condition (3.4) is replaced by the condition (2.14).

(III) If $B(x)=E_{n}$ and the conditions (3.3) and (2.14) are, respectively, replaced by the conditions

$$
\begin{aligned}
\limsup _{x \rightarrow \infty} \frac{1}{H(x, a)} \mathrm{L}\left[-\int_{a}^{x} \beta(s)\{H(x, s) k(s)[C+\right. & \left.A^{*} A\right](s) \\
& \left.\left.+\frac{1}{2} h(x, s)\left[A^{*}+A\right](s)\right\} \mathrm{d} s\right]=\infty
\end{aligned}
$$

and (2.15), then the system (1.3) is oscillatory on $[a, \infty)$.

Proof. By the definition of $G_{1}(x)$, we obtain

$$
\begin{aligned}
\mathrm{L}\left[J_{0}(x)\right] & =\mathrm{L}\left[G_{1}(x)\right]+\mathrm{L}\left[-\int_{a}^{x} \frac{h^{2}(x, s)}{H(x, s) k(s)} \beta(s) B^{-1}(s) \mathrm{d} s\right] \\
& =\mathrm{L}\left[G_{1}(x)\right]-\mathrm{L}\left[\int_{a}^{x} \frac{h^{2}(x, s)}{H(x, s) k(s)} \beta(s) B^{-1}(s) \mathrm{d} s\right] \\
& \geqslant \mathrm{L}\left[G_{1}(x)\right]-\limsup _{x \rightarrow \infty} \mathrm{L}\left[\int_{a}^{x} \frac{h^{2}(x, s)}{H(x, s) k(s)} \beta(s) B^{-1}(s) \mathrm{d} s\right]-1
\end{aligned}
$$


for sufficiently large $x$. From (3.3) and (3.4), we have

$$
\limsup _{x \rightarrow \infty} \frac{1}{H(x, a)} \mathrm{L}\left[J_{0}(x)\right]=\infty .
$$

Hence (1.1) is oscillatory on $[a, \infty)$.

The second and third parts are consequences of the first. The proof is complete.

Choosing $\beta(t) \equiv 1$ in Theorem 3.2, we obtain the following corollary.

Corollary 3.5. Let functions $H \in C(D ; \mathbb{R})$ satisfy conditions $\left(H_{1}\right)$ and $\left(H_{2}\right)$ in Theorem 2.2. If there exist functions $\phi \in C^{1}([0, \infty),(0, \infty)), h \in C\left(D_{0} ; \mathbb{R}\right)$ and $k \in C^{1}([a, \infty) ;(0, \infty))$ such that

$$
\begin{gathered}
-\frac{\partial}{\partial s}(H(x, s) k(s))=h(x, s), \quad \forall(x, s) \in D_{0}, \\
\limsup _{x \rightarrow \infty} \frac{1}{H(x, a)} \mathrm{L}\left[\int_{a}^{x}\left\{H(x, s) k(s) T(s)+J^{0}(x, s)\right\} \mathrm{d} s\right]=\infty,
\end{gathered}
$$

where

$$
v(x)=\exp \left\{-2 \int_{a}^{x} \phi(s) \mathrm{d} s\right\}, \quad T(s)=v(s)\left[-C-\phi\left(A^{*}+A\right)+\phi^{2} B-\phi^{\prime} E_{n}\right](s)
$$

and $J^{0}(x, s)$ is defined as in (2.17), then the system (1.1) is oscillatory.

Remark 3.6. If $h(t, s)$ is replaced by $h(t, s) \sqrt{H(t, s) k(s)}$ in Theorem 3.2 (as we did in Theorem 2.8) and Corollaries 3.3-3.5, we can establish similar oscillation results.

Remark 3.7. Theorem 3.2 and Corollaries 3.3-3.5 are generalizations of the analogous oscillation criterion of Kumari and Umamaheswaram [8, Theorems 2.3, 2.9] for the system (1.1). If $n=1$ and $A(x)=0$, the system (1.1) reduces to the scalar system (1.6). Note further that if we take $k(x) \equiv \beta(x) \equiv 1$, Theorem 3.2 and Corollaries 3.3-3.5 also improve and generalize the results of $\mathrm{Li}[\mathbf{1 0}]$, Philos [13] and Kamenev [7].

We remark that choices of $k(s), \beta(s), \phi(s)$ include $1, s$, etc.; while choices of $H$ include $H(t, s)=(t-s)^{\lambda}, H(x, s)=[R(x)-R(s)]^{\lambda}, H(x, s)=[\log Q(x) / Q(s)]^{\lambda}$, or

$$
H(x, s)=\left[\int_{s}^{x} \frac{1}{w(z)} \mathrm{d} z\right]^{\lambda}
$$

etc., for $x \geqslant s \geqslant a$, where $\lambda>1$ is a constant,

$$
R(x)=\int_{a}^{x} \frac{1}{u(s)} \mathrm{d} s, \quad Q(x)=\int_{t}^{\infty} \frac{1}{u(s)} \mathrm{d} s<\infty,
$$

for $x \geqslant a$, and $w \in C\left(\left[t_{0}, \infty\right), R_{+}\right)$, which satisfies $\int_{a}^{\infty} \mathrm{d} s / w(s)=\infty$.

Moreover, from Theorems 2.2, 2.8, Corollaries 2.4-2.7, Theorem 3.2 and Corollaries 3.3-3.5, we can present different explicit sufficient conditions for the oscillation of (1.1) by properly choosing the functionals $p, L$ and the functions $\beta, \phi, k$. For instance, we may choose $k(x)=1, x$, etc. 


\section{Examples}

The following example illustrates Theorem 2.8. It is easy to see that Theorem 2.3 of [8] is not applicable in our example. Furthermore, the results in $[\mathbf{1}-\mathbf{3}, \mathbf{5}, \mathbf{1 1}, \mathbf{1 5}]$ are also not applicable since $A(x) \not \equiv 0$.

Example 4.1. Consider the system (1.1) where

$$
A(x)=\left[\begin{array}{ll}
0 & 0 \\
1 & 0
\end{array}\right], \quad B(x)=\left[\begin{array}{ll}
x & 0 \\
0 & x
\end{array}\right], \quad C(x)=\left[\begin{array}{cc}
-1 / x & 0 \\
0 & 0
\end{array}\right], \quad x \geqslant 1 .
$$

Let $p[K]=\lambda_{\max }[K]$ for $K=\left(K_{i j}\right) \in \mathcal{S}, H(x, s)=(x-s)^{2}, k(s)=s, \beta(s)=s^{2}$ and $\phi(s) \equiv 0$. Then

$$
\begin{aligned}
h(x, s) & =\frac{2(x-s) s-(x-s)^{2}}{(x-s) \sqrt{s}}-\frac{(x-s)^{2} s}{(x-s) \sqrt{s}} \frac{2 s}{s^{2}}=\frac{1}{\sqrt{s}}(5 s-3 x), \\
J_{1}^{0}(x, s) & =\int_{1}^{x} \beta(s) H(x, s) k(s)\left[C+A^{*} B^{-1} A\right](s) \mathrm{d} s \\
& =\int_{1}^{x} s^{2}(x-s)^{2} s \mathrm{~d} s\left\{\left[\begin{array}{cc}
-1 / s & 0 \\
0 & 0
\end{array}\right]+\left[\begin{array}{cc}
1 / s & 0 \\
0 & 0
\end{array}\right]\right\}=\left[\begin{array}{ll}
0 & 0 \\
0 & 0
\end{array}\right], \\
J_{2}^{0}(x, s) & =\frac{1}{2} \int_{1}^{x} \beta(s) h(x, s) \sqrt{H(x, s) k(s)}\left[A^{*} B^{-1}+B^{-1} A\right](s) \mathrm{d} s \\
& =\frac{1}{2} \int_{1}^{x} s^{2} \frac{1}{\sqrt{s}}(5 s-3 x)(x-s) \sqrt{s} \mathrm{~d} s\left[\begin{array}{cc}
0 & 1 / s \\
1 / s & 0
\end{array}\right] \\
& =-\frac{1}{2} \int_{1}^{x}\left(3 x^{2} s-8 x s^{2}+5 s^{3}\right) \mathrm{d} s\left[\begin{array}{ll}
0 & 1 \\
1 & 0
\end{array}\right] \\
& =\left(-\frac{1}{24} x^{4}+\frac{3}{4} x^{2}-\frac{4}{3} x+\frac{5}{8}\right)\left[\begin{array}{ll}
0 & 1 \\
1 & 0
\end{array}\right], \\
J_{3}^{0}(x, s) & =\frac{1}{4} \int_{1}^{x} \beta(s) h^{2}(x, s) B^{-1}(s) \mathrm{d} s=\frac{1}{4} \int_{1}^{x} s^{2} \frac{1}{s}(5 s-3 x)^{2}\left[\begin{array}{cc}
1 / s & 0 \\
0 & 1 / s
\end{array}\right] \mathrm{d} s \\
& =\left(\frac{7}{12} x^{3}-\frac{9}{4} x^{2}+\frac{15}{4} x-\frac{25}{12}\right)\left[\begin{array}{ll}
1 & 0 \\
0 & 1
\end{array}\right],
\end{aligned}
$$

and

$$
\begin{aligned}
\int_{a}^{x}\{H(x, s) k(s) T(s)+ & \left.J_{0}^{0}(x, s)\right\} \mathrm{d} s \\
& =-J_{1}^{0}(x, s)-J_{2}^{0}(x, s)-J_{3}^{0}(x, s) \\
& =\left[\begin{array}{cc}
-\frac{7}{12} x^{3}+\frac{9}{4} x^{2}-\frac{15}{4} x+\frac{25}{12} & \frac{1}{24} x^{4}-\frac{3}{4} x^{2}+\frac{4}{3} x-\frac{5}{8} \\
\frac{1}{24} x^{4}-\frac{3}{4} x^{2}+\frac{4}{3} x-\frac{5}{8} & -\frac{7}{12} x^{3}+\frac{9}{4} x^{2}-\frac{15}{4} x+\frac{25}{12}
\end{array}\right] .
\end{aligned}
$$


Hence

$$
\begin{aligned}
& \limsup _{x \rightarrow \infty} \frac{1}{H(x, a)} \lambda_{\max }\left[\int_{a}^{x}\left\{H(x, s) k(s) T(s)+J_{0}^{0}(x, s)\right\} \mathrm{d} s\right] \\
& \geqslant \limsup _{x \rightarrow \infty}\left[\frac{1}{24} x^{2}\left(\frac{x}{x-1}\right)^{2}-\frac{7}{12} x \frac{x}{x-1}\right]=\infty .
\end{aligned}
$$

Thus all the assumptions of Theorem 2.8 are satisfied. By Theorem 2.8 the system (1.1) is oscillatory. Furthermore, this example illustrates that the assumption in Theorem 2.8 cannot be simplified by replacing $k(s)$ by 1 .

Example 4.2. Consider the system (1.1) where

$$
A(x)=\left[\begin{array}{ll}
0 & 0 \\
1 & 0
\end{array}\right], \quad B(x)=\left[\begin{array}{ll}
1 & 0 \\
0 & 1
\end{array}\right], \quad C(x)=\left[\begin{array}{cc}
-1 & 0 \\
0 & 0
\end{array}\right], \quad x \geqslant 0
$$

Then (1.1) is oscillatory by Corollary 2.4. In fact, choose $H(x, s)=(x-s)^{2}, k(s)=1$ and $\beta(s)=s^{2}$, we obtain

$$
\begin{aligned}
h(x, s)=2(x-s)-(x-s)^{2} \frac{2 s}{s^{2}}=\frac{2}{s}(x-s)(2 s-x) & \\
\int_{0}^{x} \beta(s) H(x, s) k(s)\left[C+A^{*} B^{-1} A\right](s) \mathrm{d} s & =\int_{0}^{x} s^{2}(x-s)^{2} \mathrm{~d} s\left\{\left[\begin{array}{ll}
-1 & 0 \\
0 & 0
\end{array}\right]+\left[\begin{array}{ll}
1 & 0 \\
0 & 0
\end{array}\right]\right\} \\
& =\left[\begin{array}{ll}
0 & 0 \\
0 & 0
\end{array}\right], \\
\frac{1}{2} \int_{0}^{x} \beta(s) h(x, s)\left[A^{*} B^{-1}+B^{-1} A\right](s) \mathrm{d} s & =\frac{1}{2} \int_{0}^{x} s^{2} \frac{2}{s}(x-s)(2 s-x) \mathrm{d} s\left[\begin{array}{ll}
0 & 1 \\
1 & 0
\end{array}\right] \\
& =-\frac{4}{3} x^{4}\left[\begin{array}{ll}
0 & 1 \\
1 & 0
\end{array}\right], \\
\frac{1}{4} \int_{0}^{x} \frac{h^{2}(x, s)}{H(x, s) k(s)} \beta(s) B^{-1}(s) \mathrm{d} s & =\frac{1}{4} \int_{0}^{x}\left(\begin{array}{ll}
2 s-x)^{2} E_{2} \mathrm{~d} s \\
& =\frac{1}{3} x^{3}\left[\begin{array}{ll}
1 & 0 \\
0 & 1
\end{array}\right]
\end{array}\right.
\end{aligned}
$$

and

$$
J_{0}(x)=\left[\begin{array}{cc}
-\frac{1}{3} x^{3} & \frac{4}{3} x^{4} \\
\frac{4}{3} x^{4} & -\frac{1}{3} x^{3}
\end{array}\right]
$$

The eigenvalues of $J_{0}(x)$ are

$$
\lambda_{1}\left[J_{0}(x)\right]=-\frac{1}{3} x^{3}+\frac{4}{3} x^{4}, \quad \lambda_{2}\left[J_{0}(x)\right]=-\frac{1}{3} x^{3}-\frac{4}{3} x^{4} .
$$


Hence

$$
\limsup _{x \rightarrow \infty} \frac{1}{H(x, a)} \lambda_{1}\left[J_{0}(x)\right]=\limsup _{x \rightarrow \infty}\left[-\frac{1}{3} x+\frac{4}{3} x^{2}\right]=\infty .
$$

Thus all the assumptions of Corollary 2.4 are satisfied. However, let $L\left[J_{0}(x)\right]=\operatorname{tr}\left[J_{0}(x)\right]$, then

$$
\limsup _{x \rightarrow \infty} \frac{1}{H(x, a)} \operatorname{tr}\left[J_{0}(x)\right]=-\limsup _{x \rightarrow \infty} \frac{2}{3} x=-\infty .
$$

In this case, Corollary 3.3 is not applicable.

The same conclusion can be made by noting that $(U(x), V(x))$, defined by

$$
U(x)=\left[\begin{array}{cc}
\sin x & \sin x \\
-\cos x & \sin x
\end{array}\right], \quad V(x)=\left[\begin{array}{cc}
\cos x & \cos x \\
0 & 0
\end{array}\right]
$$

is a non-trivial prepared solution of the system (1.1) and

$$
\operatorname{det} U(x)=\left|\begin{array}{cc}
\sin x & \sin x \\
-\cos x & \sin x
\end{array}\right|=\sin x(\sin x+\cos x)
$$

is oscillatory on $[0, \infty)$.

The following example illustrates that the results in $\S 3$ provide criteria different in nature from those in $\S 2$.

Example 4.3. Consider the system (1.1) where

$$
A(x)=\left[\begin{array}{ll}
0 & 0 \\
0 & 0
\end{array}\right], \quad B(x)=\left[\begin{array}{cc}
2 & 0 \\
0 & \frac{1}{1+x}
\end{array}\right], \quad C(x)=\left[\begin{array}{cc}
-1 & 0 \\
0 & 1
\end{array}\right], \quad x \geqslant 0 .
$$

Let $H(x, s)=(x-s)^{2}, k(s)=1, \beta(s)=s^{2}$ and $L[K]=k_{11}$, where $K=\left(k_{i j}\right) \in \mathcal{S}$. Then it is easy to see that

$$
\begin{aligned}
& h(x, s)=2(x-s)-(x-s)^{2} \frac{2 s}{s^{2}}=\frac{2}{s}(x-s)(2 s-x), \\
\mathrm{L}\left[J_{1}^{0}(x)\right]= & \mathrm{L}\left[\int_{0}^{x} \beta(s) H(x, s) k(s)\left[C+A^{*} B^{-1} A\right](s) \mathrm{d} s\right] \\
= & \mathrm{L}\left\{\int_{0}^{x} s^{2}(x-s)^{2}\left[\begin{array}{cc}
-1 & 0 \\
0 & 1
\end{array}\right] \mathrm{d} s\right\}=-\frac{1}{30} x^{5} \\
\mathrm{~L}\left[J_{2}^{0}(x)\right]= & \mathrm{L}\left[\frac{1}{2} \int_{0}^{x} \beta(s) h(x, s)\left[A^{*} B^{-1}+B^{-1} A\right](s) \mathrm{d} s\right]=0
\end{aligned}
$$

and

$$
\begin{aligned}
\mathrm{L}\left[J_{3}^{0}(x)\right] & =\mathrm{L}\left[\frac{1}{4} \int_{0}^{x} \frac{h^{2}(x, s)}{H(x, s) k(s)} \beta(s) B^{-1}(s) \mathrm{d} s\right] \\
& =\mathrm{L}\left\{\frac{1}{4} \int_{0}^{x} \frac{4}{s^{2}}(x-s)^{2}(2 s-x)^{2} \frac{1}{(x-s)^{2}} s^{2}\left[\begin{array}{cc}
\frac{1}{2} & 0 \\
0 & s+1
\end{array}\right] \mathrm{d} s\right\}=\frac{1}{6} x^{3} .
\end{aligned}
$$


Hence

$$
\mathrm{L}\left[J^{0}(x)\right]=-\left\{\mathrm{L}\left[J_{1}^{0}(x)\right]+\mathrm{L}\left[J_{2}^{0}(x)\right]+\mathrm{L}\left[J_{3}^{0}(x)\right]\right\}=\infty .
$$

Thus all the assumptions of Corollary 3.3 are satisfied. By Corollary 3.3 the system (1.1) is oscillatory. However, in this example, we have $\operatorname{tr}[C(x)]=0$.

Example 4.4. Consider the system (1.1) where

$$
A(x)=\left[\begin{array}{ll}
0 & 0 \\
0 & 0
\end{array}\right], \quad B(x)=\left[\begin{array}{ll}
2 & 0 \\
0 & \frac{1}{1+x}
\end{array}\right], \quad C(x)=\left[\begin{array}{cc}
-\frac{1}{2}+\cos x & 0 \\
0 & -\frac{1}{2}-\cos x
\end{array}\right] .
$$

Let $H(x, s)=(x-s)^{2}, k(s)=1, \beta(s)=s^{2}$ and $L[K]=\operatorname{tr}[K]$. Then it is easy to see that

$$
\begin{gathered}
h(x, s)=2(x-s)-(x-s)^{2} \frac{2 s}{s^{2}}=\frac{2}{s}(x-s)(2 s-x), \\
\operatorname{tr}\left[J_{1}^{0}(x)\right]=\operatorname{tr}\left[\int_{0}^{x} \beta(s) H(x, s) k(s)\left[C+A^{*} B^{-1} A\right](s) \mathrm{d} s\right] \\
=\operatorname{tr}\left\{\int_{0}^{x} s^{2}(x-s)^{2}\left[\begin{array}{cc}
-\frac{1}{2}+\cos s & 0 \\
0 & -\frac{1}{2}-\cos s
\end{array}\right] \mathrm{d} s\right\}=-\frac{1}{30} x^{5}, \\
\operatorname{tr}\left[J_{2}^{0}(x)\right]=\operatorname{tr}\left[\frac{1}{2} \int_{0}^{x} \beta(s) h(x, s)\left[A^{*} B^{-1}+B^{-1} A\right](s) \mathrm{d} s\right]=0,
\end{gathered}
$$

and

$$
\begin{aligned}
\operatorname{tr}\left[J_{3}^{0}(x)\right] & =\operatorname{tr}\left[\frac{1}{4} \int_{0}^{x} \frac{h^{2}(x, s)}{H(x, s) k(s)} \beta(s) B^{-1}(s) \mathrm{d} s\right] \\
& =\operatorname{tr}\left\{\int_{0}^{x}(2 s-x)^{2}\left[\begin{array}{cc}
\frac{1}{2} & 0 \\
0 & s+1
\end{array}\right] \mathrm{d} s\right\}=x^{4}-\frac{1}{2} x^{3} .
\end{aligned}
$$

Hence, the trace of $J^{0}(x)$ in Corollary 3.3 is

$$
\operatorname{tr}\left[J^{0}(x)\right]=-\left\{\operatorname{tr}\left[J_{1}^{0}(x)\right]+\operatorname{tr}\left[J_{2}^{0}(x)\right]+\operatorname{tr}\left[J_{3}^{0}(x)\right]\right\}=\infty .
$$

Thus all the assumptions of Corollary 3.3 are satisfied. By Corollary 3.3 the system (1.1) is oscillatory.

Acknowledgements. Q.Y. thanks Professor James S. W. Wong and Professor Yun Tang for their helpful comments in this research. The author was supported by the Science Foundation of Guangxi Province of China (no. 0236012).

\section{References}

1. G. J. Butler, L. H. Erbe And A. B. Mingarelli, Riccati techniques and variational principle in oscillatory theory for linear systems, Trans. Am. Math. Soc. 303 (1987), 263-282.

2. R. Byers, B. J. Harris And M. K. KWong, Weighted means and oscillation of secondorder matrix differential equations, J. Diff. Eqns 61 (1986), 164-177. 
3. L. H. Erbe, Q. Kong And S. RuAn, Kamenev-type theorems for second-order matrix differential systems, Proc. Am. Math. Soc. 117 (1993), 957-962.

4. P. Hartman, On non-oscillatory linear differential equations of second order, Am. J. Math. 74 (1952), 389-400.

5. P. HARTMAN, Oscillation criteria for self-adjoint second-order differential systems and 'principal sectional curvatures', J. Diff. Eqns 34 (1979), 326-338.

6. R. A. Horn And C. R. Johnson, Matrix analysis (Cambridge University Press, 1985).

7. I. V. KAMENEv, An integral test for conjugacy for linear differential equations, Mat. Zametki 23 (2) (1978), 249-251 (in Russian).

8. I. S. Kumari AND S. Umamaheswaram, Oscillation criteria for linear matrix Hamiltonian systems, J. Diff. Eqns 165 (2000), 165-174.

9. W. Leighton, On self-adjoint differential equations of second order, J. Lond. Math. Soc. 27 (1952), 37-47.

10. H. J. LI, Oscillation criteria for second-order linear differential equations, J. Math. Analysis Applic. 194 (1995), 217-234.

11. F. Meng, J. WAng And Z. Zheng, A note on Kamenev type theorems for second-order matrix differential systems, Proc. Am. Math. Soc. 126 (1998), 391-395.

12. CH. G. Philos, On a Kamenev's integral criterion for oscillation of linear differential equations of second order, Utilias Math. 24 (1983), 277-289.

13. CH. G. Philos, Oscillation theorems for linear differential equations of second order, Arch. Math. 53 (1989), 483-492.

14. W. T. REID, Sturmian theory for ordinary differential equations, Applied Mathematical Science, vol. 31 (Springer, 1980).

15. Q. WANG, Oscillation criteria for second-order matrix differential systems, Arch. Math. 76 (2001), 385-390.

16. A. Wintner, A criteria of oscillatory stability, Q. Appl. Math. 7 (1949), 115-117.

17. J. S. W. Wong, On Kamenev-type oscillation theorems for second-order differential equations with damping, J. Math. Analysis Applic. 258 (2001), 244-257.

18. Q. G. YAng, R. MAThSEn AND S. M. ZhU, Oscillation theorems for self-adjoint matrix Hamiltonian systems, J. Diff. Eqns 190 (2003), 306-329. 